\section{Xe-MRI and QCT from a subject with COPD}

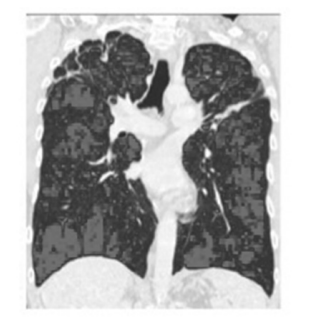

QCT emphysema density map

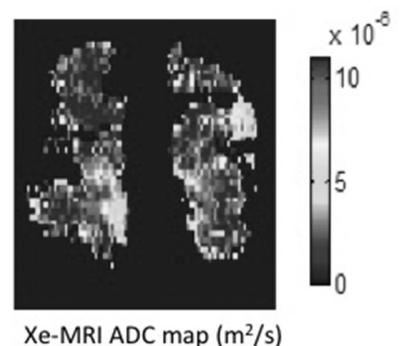

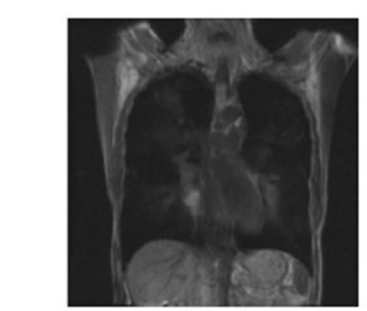

Xe-MRI ventilation co-registered with conventional MRI

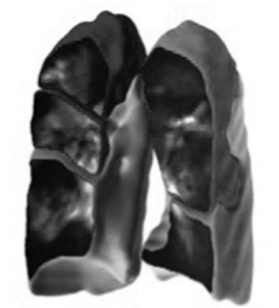

Xe-MRI lobar ventilation

Abstract S19 Figure 1

diffusion-weighted images $\left(b=20.855 \mathrm{sec} / \mathrm{cm}^{2}\right)$. Percentage predicted PFT results were established for each participant.

Whole lung and lobar QCT-derived metrics for emphysema and bronchial wall thickness were calculated from percentage of lung tissue with density of $<-950 \mathrm{HU}$ and Pi10 (the square root of wall area for an airway with lumen perimeter of $10 \mathrm{~mm}$ ), respectively.

Pearson's correlation coefficients were used to evaluate the relationship between whole lung and lobar imaging measures and PFTs.

Results Xe-MRI whole lung average ADC showed significant correlation with: whole lung QCT percentage emphysema $(r=0.79$, $\mathrm{p}=0.001)$, whole lung Pi10 $(r=0.68, \mathrm{p}<0.05)$, percentage predicted functional residual capacity (FRC) $(r=0.635, \mathrm{p}<0.05)$ and demonstrated significant negative correlation with percentage predicted TLCO $(r=-0.81, \mathrm{p}<0.001)$. Whole lung QCT percentage emphysema showed a similar significant negative correlation with percentage predicted TLCO $(r=-0.80, \mathrm{p}<0.001)$. Xe-MRI lobar percentage ventilated volume showed significant correlation with lobar QCT percentage emphysema $(r=-0.51, \mathrm{p}<<0.001)$. The QCT-derived metrics, percentage emphysema and Pi10 demonstrated significant correlation on a whole lung $(r=0.75, \mathrm{p}<$ $0.015)$ and lobar basis $(r=0.29, \mathrm{p}<0.015)$.

Conclusion This study demonstrates excellent correlation between Xe-MRI, QCT-derived metrics and PFTs in COPD. New quantitative whole lung and lobar functional imaging parameters have been derived that may be of value when assessing patients with COPD for regional treatment and in trialling new therapies. Although further investigation is required, this may represent the first integrated regional lung imaging technique linked to pulmonary functional outcomes.

\title{
S20 18F-FLUORODEOXYGLUCOSE (18FDG) PET PULMONARY IMAGING: COMPARATIVE METHODOLOGY IN COPD PATIENTS
}

${ }^{1} \mathrm{G}$ Choudhury, ${ }^{1} \mathrm{~A}$ Fletcher, ${ }^{1} \mathrm{M}$ Connell, ${ }^{2} \mathrm{~B}$ Whitcher, ${ }^{1} \mathrm{~S}$ Fergusson, ${ }^{1} \mathrm{~T}$ Clark, ${ }^{2} \mathrm{~B}$ Vennart, ${ }^{2}{ }^{2}$ Kilty, ${ }^{1} E$ VanBeek, 'W MacNee. 'Queens Medical Research Institute, Edinburgh, UK; ${ }^{2}$ Pfizer Inc, USA

10.1136/thoraxjnl-2014-206260.26

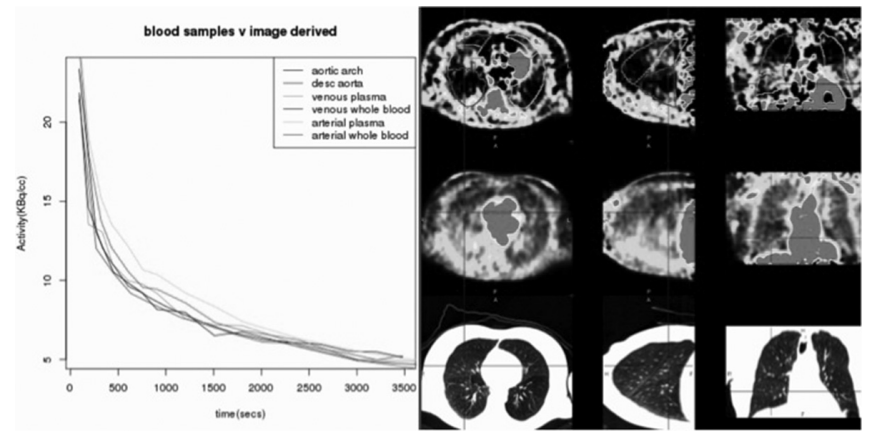

Abstract S20 Figure 1 Shows an example of time activity curves from arterial, venous and image derived techniques (on left) and (on right) a Patlak image from venous plasma slope (1st row), intercept (2nd row) and CT (3rd row)

Introduction ${ }^{18}$ FDG PET/CT imaging may be a useful tool to study COPD and lung inflammation; however the optimal protocol for this imaging biomarker has yet to be established.

Method We aimed to develop a combined ${ }^{18}$ FDG-PET/CT imaging protocol optimised to quantify lung inflammation. Six patients with moderate-to-severe COPD underwent dynamic ${ }^{18}$ FDG-PET imaging combined with blood sampling (both arterial and venous over $60 \mathrm{~min}$ ) to determine the localised plasma activity time curve. High resolution CT (HRCT) was utilised to segmentate the lung and determine areas of emphysema. 3 sets of comparative input functions were analysed (arterial, venous and image derived arterial input functions). ${ }^{18}$ FDG kinetics was fitted using the Patlak method.

Results Similar results were obtained using time activity curves from all three input functions. The arterial input was always found to be slightly higher than the others (Figure 1). Patlak analysis of the time-activity curves for each of the CT derived lung lobes allowed generation of images of slope (influx constant Ki) and intercept (initial volume of distribution) (Figure 1). The acquisition of HRCT co-registered to FDG-PET allows more accurate demarcation and quantification of FDG in emphysematous areas of the lung. Attempt to improve the signal by excluding voxels without COPD tissue $(-935$ to $-300 \mathrm{HU})$ has been undertaken as well. The reproducibility of this technique is currently being studied where 20 patients are being scanned twice 4 weeks apart and compared to a baseline scan from 5 healthy controls.

Conclusion ${ }^{18}$ FDG PET/CT imaging has the potential to be a non-invasive biomarker of lung inflammation in COPD.

\section{S21 CULTURE INDEPENDENT IDENTIFICATION OF BACTERIAL COMMUNITIES IN THE RESPIRATORY TRACT OF PATIENTS WITH COPD, HEALTHY NON-SMOKERS AND HEALTHY SMOKERS}

${ }^{1} \mathrm{GG}$ Einarsson, ${ }^{2} \mathrm{~A}$ Walker, ${ }^{3} \mathrm{MM}$ Tunney, ${ }^{4} \mathrm{JS}$ Elborn. ${ }^{1} \mathrm{CF}$ and Airways Microbiology Research Group, Queen's University Belfast, Belfast, UK; ${ }^{3}$ School of Pharmacy, Queen's University Belfast, Belfast, UK; ${ }^{4}$ School of Medicine, Dentistry and Biomedical Sciences, Queen's University Belfast, Belfast, UK; ${ }^{2}$ Wellcome Trust Sanger Institute, Wellcome Trust Genome Campus, Hinxton, Cambridge, UK

\subsection{6/thoraxjn-2014-206260.27}

Introduction and aims The role bacteria play in the development and progression of Chronic Obstructive Pulmonary Disease (COPD) is unclear. We used culture-independent methods to describe differences and/or similarities in microbial communities 